\title{
Calpain 6 inhibits autophagy in inflammatory environments: A preliminary study on myoblasts and a chronic kidney disease rat model
}

\author{
YUE YUE ZHANG, LI JIE GU, NAN ZHU, LING WANG, MIN CHAO CAI, \\ JIE SHUANG JIA, SHU RONG and WEI JIE YUAN \\ Division of Nephrology, Shanghai General Hospital, Shanghai Jiaotong University \\ School of Medicine, Shanghai 200080, P.R. China
}

Received March 16, 2021; Accepted June 30, 2021

DOI: $10.3892 /$ ijmm.2021.5027

\begin{abstract}
A non-classical calpain, calpain 6 (CAPN6), can inhibit skeletal muscle differentiation and regeneration. In the present study, the role of CAPN6 in the regulation of the autophagy of myoblasts in vitro was investigated. The underlying molecular events and the CAPN6 level in atrophic skeletal muscle in a rat model of chronic kidney disease (CKD) were also investigated. In vitro, CAPN6 was overexpressed, or knocked down, in rat L6 myoblasts to assess autophagy and related gene expression and co-localization. Subsequently, myoblasts were treated with a mixture of cytokines, and relative gene expression and autophagy were assessed. A rat model of CKD for muscle atrophy was established, and blood chemical level and the expression of CAPN6 in muscle were assessed. The data revealed that the knockdown of CAPN6 in rat myoblasts resulted in increased microtubule-associated protein 1 light chain 3 (LC3) levels, while its overexpression decreased LC3 levels and impaired autophagy. Additionally, it was observed that the co-localization of mammalian target of rapamycin (mTOR) and lysosomal-associated membrane protein 1 (LAMP1), a lysosomal marker, proteins was increased. In addition, mTOR, Raptor and $\alpha$-tubulin (a marker of microtubules) increased in the CAPN6 overexpression group. However, inflammatory cytokines, such as interleukin (IL)-6, tumor necrosis factor (TNF)- $\alpha$, interferon (INF)- $\gamma$ and lipopolysaccharides upregulated CAPN6 expression, inhibited L6 myoblast autophagy and stabilized mTOR activity. Furthermore, the animal model successfully mimicked human
\end{abstract}

Correspondence to: Dr Wei Jie Yuan or Dr Shu Rong, Division of Nephrology, Shanghai General Hospital, Shanghai Jiaotong University School of Medicine, 85 Wujin Road, Shanghai 200080, P.R. China

E-mail: ywj4169@163.com

E-mail: goldenxc@126.com

Key words: chronic kidney disease, calpain 6, autophagy, mTOR, inflammation, muscle atrophy disease as regards an increase in body weight, and a reduction in muscle mass, cross-sectional area and blood biomarker concentrations; a slight increase in CAPN6 mRNA and protein levels in muscles was observed. Finally, the data of the present study suggested that CAPN6 reduced autophagy via the maintenance of mTOR signaling, which may play a role in CKD-related muscle atrophy. However, future studies are required to determine whether CAPN6 may be used as an intervention target for CKD-related skeletal muscle atrophy.

\section{Introduction}

Chronic kidney disease (CKD) is associated with early-age mortality, a decrease in the quality of life of affected individuals and increased health care costs (1). CKD alters complex metabolic processes that affect muscular homeostasis, resulting in a loss in muscle mass and, ultimately, in muscle atrophy (2,3). Approximately 16 to $54 \%$ of patients with CKD are malnourished (4). The term protein-energy wasting (PEW) has been suggested to describe this syndromic uremic malnutrition (5) and to denote the concurrent losses in protein and energy storage (6), while skeletal muscle atrophy is the main PEW characteristic. As CKD progresses, the risk of PEW increases (7), ultimately increasing the risk of mortality (8). Therefore, the identification of the PEW-inducing mechanisms and the exploration of possible interventions may aid in the provision of practical and effective treatment approaches for the clinical prognosis of patients with CKD.

Autophagy and mitophagy can play an important role in CKD-related muscle atrophy (9). Autophagy is a part of the lysosomal degradation pathway and its main function is to remove unnecessary or dysfunctional components from cells, playing an important role in the maintenance of cell homeostasis (10-12). Demarchi et al (13) found that autophagy was damaged in cells with a defective calpain small subunit. In addition, calpain-6 (CAPN6) can antagonize the effects of other classical forms of calpain (14), indicating that CAPN6 can inhibit autophagy.

CAPN6 is a non-classical calpain (cannot degrade proteins) and is mainly expressed in fetal muscle and the placenta. CAPN6 expression is reduced after birth, but is upregulated 
in various tumor tissues (15). A previous study revealed that CAPN6 deficiency promoted skeletal muscle development and regeneration, whereas it suppressed differentiation (16). Another study, involving DNA microarray cluster analysis, revealed that CAPN6 expression was upregulated in the sera of patients with type 2 diabetic nephropathy (17). The Gene Expression Omnibus (GEO) database on CAPN6 expression in patients with CKD revealed that CAPN6 expression was higher in CKD renal tissues than in control tissues (https://www.ncbi. nlm.nih.gov/geo/query/acc.cgi?acc=GSE48944) (18). However, Andrique et al (19) reported that CAPN6 promoted autophagy and prevented senescence in hypoxic environments to regulate sarcoma stem-cell fate and tumor progression. Therefore, it is important to understand the role of CAPN6 and its regulatory effects on autophagy in CKD.

Autophagy can be regulated by the canonical mammalian target of rapamycin (mTOR)/Unc-51 like autophagy activating kinase 1 (ULK1/2) and 5'AMP-activated protein kinase (AMPK) pathways (20). mTOR can form the mTOR complex 1 (mTORC1) - mTOR, Raptor, mammalian lethal with SEC13 protein 8 (mLST8) and proline-rich Akt substrate 40 (PRAS40) (21-23), and plays an important role in negative autophagy regulation $(24,25)$, particularly in the initial phase. Additionally, mTORC1 can suppress the formation and fusion of the autophagic compartment with lysosomes (26). Liu et al (27) reported that rapamycin (mTOR inhibitor) was able to reduce CAPN6 protein levels, indicating that the Akt signaling pathway participated in CAPN6 regulation through mTOR. However, mTOR did not affect the stability and degradation of CAPN6 protein. Thus, further studies are required, as regards the regulation of autophagy by CAPN6 in CKD-related muscle atrophy, in order to assist clinicians in effectively delaying, or even having control over this issue.

IL-6, TNF- $\alpha$ and IFN- $\gamma$ expression levels are upregulated in patients with CKD and dialysis, and are increased in parallel with lipopolysaccharide (LPS) $(28,29)$. Thomas et al (30) revealed that the combination of IL- 6 , TNF- $\alpha$, IFN- $\gamma$ and LPS produced a time-dependent decrement in phosphorylated tyrosine levels of both IRS-1 and insulin receptor, playing an important role in CKD-related muscle wasting. In consideration of these results, the present study aimed to elucidate the underlying mechanisms through cytokines may increase CAPN6 expression in muscles.

Therefore, the role of CAPN6 in the regulation of autophagy in myoblasts in vitro and the underlying molecular events were investigated in the present study. In addition, the CAPN6 levels in atrophic skeletal muscle in a rat model of CKD were evaluated, in order to primarily explore the role of CAPN6 in skeletal muscle atrophy in CKD.

\section{Materials and methods}

Animals and animal experiments. All animal experiments were approved by the Animal Care and Use Committee of Shanghai Jiao Tong University (Shanghai, China) and followed the Guidelines of the Care and Use of Laboratory Animals issued by the Chinese Council on Animal Research (project no. 2015DW001). Specifically, male Sprague-Dawley rats (200-250 g body weight) were purchased from the Chinese Academy of Sciences, bred, and maintained at the Animal
Center Laboratory of Shanghai General Hospital. A total of 2 mice were housed per cage, under controlled temperature $\left(22-25^{\circ} \mathrm{C}\right)$ and humidity $(70 \%)$ conditions, along with a $12-\mathrm{h}$ light/dark cycle, and the provision of free access to water. Rats were randomly assigned to either the 5/6 nephrectomized or the sham-operated control group ( $n=8$ per group), and received surgical procedures according to a previous study (31). Briefly, the rats were anesthetized by an intraperitoneal injection of sodium pentobarbital $(40 \mathrm{mg} / \mathrm{kg})$ and received the 5/6 nephrectomy by surgical resection of the upper and lower thirds of the left kidney followed by right nephrectomy 7 days later. In the control group, only a sham operation was performed. During the experimental period, the animals were monitored two to three times per week for potential signs of suffering, mainly a weight loss $>20 \%$ and significant changes in their behavior, mobility or body posture. In the case that the rats met one of these criteria, euthanasia would have been warranted on the same day in order to prevent further suffering. Animals were kept for 6 months. One CKD rat died in the late experimental period, and all control rats survived, similar to previous studies $(32,33)$. Following adequate anesthesia with sodium pentobarbital $(40 \mathrm{mg} / \mathrm{kg})$ intraperitoneal injection, the bodyweight of each rat was recorded and blood samples were then collected through cardiac puncture, in order to measure creatinine, blood urea nitrogen (BUN), albumin (Alb), total cholesterol (TC) and triglyceride (TG) serum levels. Gastrocnemius muscle samples were collected and used to measure muscle wet weight, perform reverse transcription-quantitative PCR (RT-qPCR) and western blot analyses. Finally, the rats were sacrificed by decapitation immediately after the experimental procedure when the rats were still under deep anesthesia. Death was further confirmed by checking for the onset of rigor mortis (34).

Hematoxylin and eosin (H\&E) staining. Gastrocnemius muscle samples were fixed in $4 \%$ freshly made paraformaldehyde and processed for embedding in paraffin and sectioning. Tissue sections at a thickness of $4 \mu \mathrm{m}$ were stained with $\mathrm{H} \& \mathrm{E}$ (Wuhan Servicebio Technology Co., Ltd.) at room temperature for 1-3 min, using standard procedures and observed under a light microscope (DFC550; Leica Microsystems, Inc.). Cross-sectional areas (CSAs) were analyzed using Image-Pro Plus 6.0 software (Media Cybernetics, Inc.) after counting at least 100 muscle fibers per muscle.

Cells and cell culture. Rat L6 myoblasts were purchased from The Cell Bank of Type Culture Collection of the Chinese Academy of Sciences (GNR 4), and were passaged according to previous studies $(35,36)$. L6 cells were seeded in 6-well plates ( $1 \times 10^{5}$ cells per well) in Dulbecco's modified Eagle's medium (DMEM; Gibco; Thermo Fisher Scientific, Inc.), supplemented with $10 \%$ fetal bovine serum [(FBS); (Gibco; Thermo Fisher Scientific, Inc.)] and $1 \%$ penicillin/streptomycin. Afterwards, the L6 myoblasts were incubated in a humidified incubator with $5 \% \mathrm{CO}_{2}$ at $37^{\circ} \mathrm{C}$ overnight. Then cell culture medium was replaced with DMEM containing only $2 \%$ horse serum to initiate differentiation for $48 \mathrm{~h}$.

To mimic the conditions of CKD in rats and patients in vivo, L6 myoblasts were exposed to four different inflammatory agents [2 ng/ml IL-6; (400-06, Peprotech), 2 ng/ml TNF- $\alpha$ 
(400-14, Peprotech), 2 ng/ml INF- $\gamma$ (400-20, Peprotech) and $10 \mathrm{ng} / \mathrm{ml}$ LPS (SMB00610, Sigma-Aldrich; Merck KGaA)] according to a previous study (30), and the CAPN6 expression levels were evaluated.

Cell transfection. Plasmids carrying CAPN6 shRNA (hU6-MCS-SV40-Neomycin- CAPN6 RNAi and $\mathrm{CAPN}^{-1-}$ ) and shRNA negative control were purchased from GeneChem, Inc. and the CAPN6 CRISPR activation plasmid (cat. no. sc-437325-ACT) and the control CRISPR activation plasmid (cat. no. sc-437275) were obtained from Santa Cruz Biotechnology, Inc. Plasmids carrying Raptor shRNA (phb-u6-mcs-pgk-puro-Raptor RNAi and Raptor ${ }^{-/}$) and shRNA negative control were purchased from Hanbio Biotechnology Co., Ltd. For cell transfection, L6 cells were grown overnight and transiently transfected at room temperature using OptiMEM medium with Lipofectamine $2000^{\circledR}$ (Invitrogen; Thermo Fisher Scientific, Inc.), according to the manufacturer's instructions. The final concentration of the plasmids was $2 \mathrm{ng} / \mu \mathrm{l}$. Following $6 \mathrm{~h}$ of transfection at $37^{\circ} \mathrm{C}$, treatments were applied to the cells.

Autophagy assay. Red and green fluorescent protein-tagged microtubule-associated protein 1 light chain 3 (RFP-GFP-LC3) adenoviral vectors (Hanbio Biotechnology Co., Ltd.) was used to transfect the L6 cells for the measurement of autophagy levels, using OptiMEM medium with Lipofectamine $2000^{\circledR}$ (Invitrogen; Thermo Fisher Scientific, Inc.), according to the manufacturer's protocol. The final concentration of the plasmids was $10 \mu \mathrm{l} / \mathrm{ml}$. The transfection efficiency was inspected with a Leica Microsystems GmbH confocal microscope (Leica DM6000B). The RFP-GFP-LC3 levels in transfected cells was also visualized using a Leica Microsystems $\mathrm{GmbH}$ confocal microscope (Leica TCS SP8) and quantified using Image-Pro plus 6.0 (Media Cybernetics, Inc.).

$R T-q P C R$. Total cellular RNA was isolated from the tissues and cells using TRIzol ${ }^{\circledR}$ reagent (cat. no. 15596-026; Invitrogen; Thermo Fisher Scientific, Inc.) and reverse transcribed into cDNA using the High Capacity cDNA Reverse Transcription kit (cat. no. K1622; Thermo Fisher Scientific, Inc.) according to the manufacturer's protocols. qPCR was performed using SYBR-Green (cat. no. F-415XL; Thermo Fisher Scientific, Inc.) in an ABI PRISM Sequence Detector System 7300 (Applied Biosystems; Thermo Fisher Scientific, Inc.). Conditions were set to initial $95^{\circ} \mathrm{C}$ for $10 \mathrm{~min}$, then 40 cycles of $95^{\circ} \mathrm{C}$ for $15 \mathrm{sec}$ and $60^{\circ} \mathrm{C}$ for $60 \mathrm{sec}$. Each experiment was performed in triplicate and repeated three times. The CAPN6 relative mRNA expression levels were calculated using the $2^{-\Delta \Delta C q}$ method (37). The primers used were as follows: CAPN6 forward, 5'-TGT TTGGCTGTTCAGGAGTC-3' and reverse, 5'-TGGGAAGCA AGTCGTCAATC-3'; and glyceraldehyde 3-phosphate dehydrogenase (GAPDH) forward, 5'-GTCGGTGTGAACGGA TTTG-3' and reverse, 5'-TCCCATTCTCAGCCTTGAC-3'.

Western blot analysis. Tissue and cell samples were pulverized and lysed in the radioimmunoprecipitation assay (RIPA) buffer (Beyotime Institute of Biotechnology) at $4^{\circ} \mathrm{C}$ for $2 \mathrm{~h}$. The lysates were centrifuged at $10,000 \mathrm{xg}$ at $4^{\circ} \mathrm{C}$ for $10 \mathrm{~min}$ to collect the supernatant into separate tubes. The quality and concentration of protein were detected using a NanoDrop spectrophotometer (Thermo Fisher Scientific, Inc.). Protein samples $(20 \mu \mathrm{g})$ were separated with sodium dodecyl sulfate-polyacrylamide gel electrophoresis (SDS-PAGE) and electro-transferred onto nitrocellulose membranes (EMD Millipore). The membranes were incubated in 5\% skim milk in phosphate-buffered saline (PBS)-Tween 20 (PBS-T) at room temperature for $1 \mathrm{~h}$, and subsequently with primary antibodies at $4^{\circ} \mathrm{C}$ overnight. The following primary antibodies used were purchased from Cell Signaling Technology, Inc.: anti-LC3 (1:1,000, cat. no. 2775), GAPDH (1:2,000, cat. no. 5174), phosphorylated (p-)eukaryotic translation initiation factor $4 \mathrm{E}$ binding protein 1 (4EBP-1; 1:1,000, cat. no. 2855), 4EPB-1 (1:1,000, cat. no. 9452); p-p70s6k1 (1:1,000, cat. no. 9234), p70s6k1 (1:1,000, cat. no. 9202) and (mTOR; 1:1,000, cat. no. 2983). Anti- CAPN6 (1:1,000, cat. no. ab76974) and Raptor (1:3,000, cat. no. ab40768) antibodies from Abcam were also used. GAPDH antibody was used as a loading control for the muscle tissues. Afterwards, the membranes were washed three times with PBS-T, then incubated with horseradish peroxidase-conjugated secondary anti-rabbit IgG (1:1.000, cat. no. A0208; Beyotime Institute of Biotechnology), or anti-mouse $\operatorname{IgG}$ (1:1.000, cat. no. A0216; Beyotime Institute of Biotechnology), for $1 \mathrm{~h}$ at room temperature. Positive protein bands were visualized using an enhanced chemiluminescence (ECL) substrate kit (cat. no. WBKLS0100; EMD Millipore), exposed to X-ray films and quantified using Tanon-5200 software (Tanon Science \& Technology Co., Ltd.).

Immunofluorescence staining. First, cell slides were washed with PBS $(0.02 \mathrm{M})$ to remove the culture medium, and fixed with $4 \%$ formaldehyde for $30 \mathrm{~min}$. Subsequently, the slides were washed three times for 3 min each with PBS (0.02 M) prior to permeabilization with Triton X-100 (0.5\%) for $10 \mathrm{~min}$. Consequently, the slides were washed three times with PBS $(0.02 \mathrm{M})$, for 3 min each. Cells were blocked with $1 \%$ BSA for $1 \mathrm{~h}$ and washed with PBS $(0.02 \mathrm{M})$ for $3 \mathrm{~min}, 3$ times. Diluted primary antibodies were added drop by drop to each cell slide, and the slides were then placed in a wet box and incubated at $4^{\circ} \mathrm{C}$ overnight. Primary antibodies used in this experiment included Raptor (1:200, cat. no. ab5454; Abcam), mTOR (1:200, cat. no. 2983; Cell Signaling Technology, Inc.), lysosomal-associated membrane protein 1 (LAMP1; 1:500, cat. no. sc-20011; Santa Cruz Biotechnology, Inc.), $\alpha$-tubulin (1:1,000, cat. no. 3873; Cell Signaling Technology, Inc.). A secondary antibody was then added in a dropwise manner, and the slides were placed into a wet box and incubated at $20^{\circ} \mathrm{C}$ for $1 \mathrm{~h}$. The secondary antibodies used, included goat anti-rabbit IgG (FITC,1:1,000, ab6717) and goat anti-mouse IgG (TR,1:1000, ab5766), purchased from Abcam. DAPI (1:500, Beyotime Institute of Biotechnology) was used for nuclei staining at room temperature for $1 \mathrm{~min}$. The slides were stored at $-20^{\circ} \mathrm{C}$ until further analysis. Images were recorded using a fluorescence microscope (Olympus Corporation). A total of five randomly selected fields of each slide were selected and analyzed using Image-Pro plus 6.0 software (Media Cybernetics, Inc.).

Statistical analysis. Data are presented as the mean \pm standard deviation (SD) and were statistically analyzed using SPSS 17.0 
A
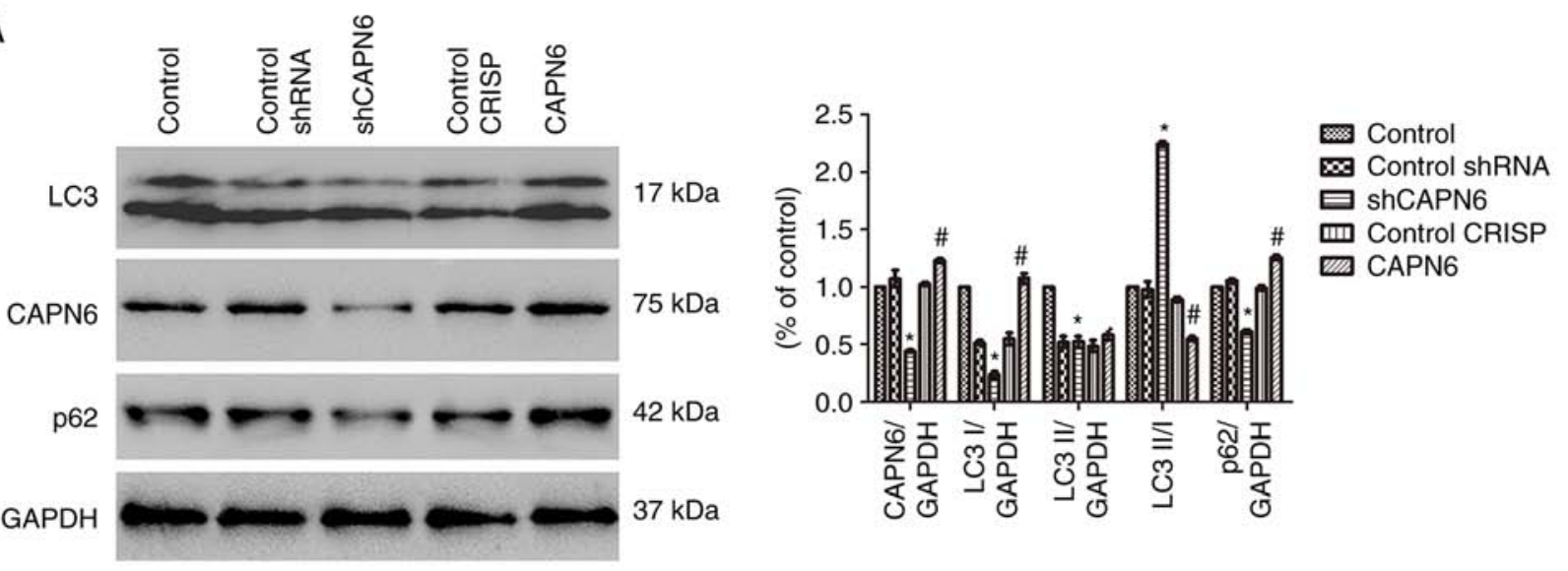

B
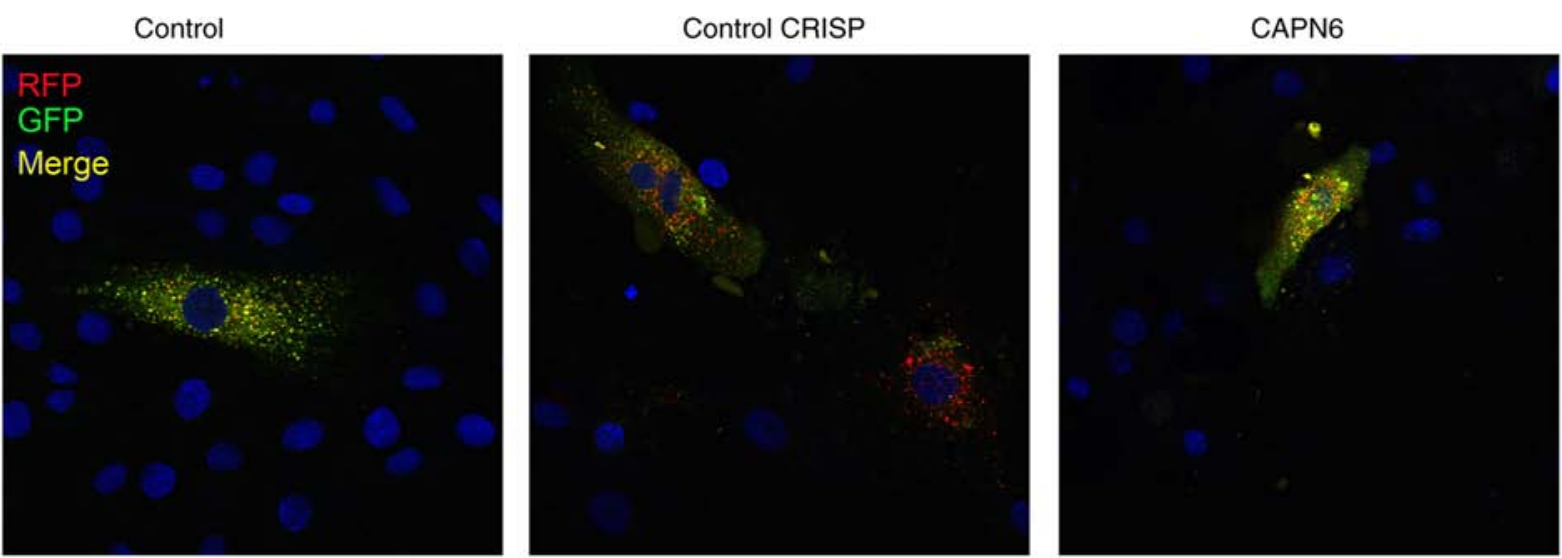

Figure 1. CAPN6 decreases autophagy in rat L6 myoblasts. (A) L6 myoblasts were grown and transiently transfected with CAPN6 shRNA and cDNA, respectively, then subjected to western blot analysis of CAPN6, LC3 and p62. The graph summarizes the western blotting results. *P $<0.05$ in comparison with the control group. ${ }^{\#} \mathrm{P}<0.05$ in comparison with the shCAPN6 group. (B) Immunofluorescence: L6 myoblasts were grown and transiently transfected with a control and CAPN6 cDNA respectively, then subjected to immunofluorescence analysis of GFP- RFP-LC3 (magnification, x630). CAPN6, Calpain 6; LC3, microtubule-associated protein 1 light chain 3; GFP, green fluorescent protein; RFP, red fluorescent protein.

statistical software (SPSS, Inc.). In particular, data with a normal distribution were analyzed using one-way analysis of variance (ANOVA), followed by pairwise comparisons with the Tukey's test. Data with unequal and non-parametric features were analyzed using ANOVA on ranks followed by the Dunn's method for pairwise comparisons. Comparisons between two groups were analyzed with an independent Student's t-test. $\mathrm{P} \leq 0.05$ was considered to indicate a statistically significant difference.

\section{Results}

CAPN6 rat L6 myoblast autophagy reduction and the possible underlying mechanisms. To explore the involvement of CAPN6 in the regulation of myoblast autophagy, the expression of the autophagy-related genes, LC3 and p62, in rat L6 myoblast cells were examined. First, CAPN6 overexpression and knockdown was induced in L6 cells (Fig. S1). CAPN6 shRNA decreased CAPN6 protein levels by $55.75 \%$ and increased the ratio of LC3II/I by $124.815 \%$, whereas CAPN6 cDNA increased the CAPN6 levels by $23.6 \%$ and decreased the ratio of LC3II/I by $45.03 \%$ (Fig. 1). This was also confirmed by p62, a cargo protein degraded inside autolysosomes, protein level detection. The level of p62 decreased when CAPN6 expression decreased (Fig. 1).
To examine the autophagic flux status, L6 myoblasts were transfected with RFP-GFP-LC3 plasmid, according to a previous study (38). The green fluorescent protein (GFP) level is quenched in the lysosomal environment and the red fluorescent protein (RFP) signal is more stable in the acidic environment according to a previous study (39). Thus, the autophagosomes and autolysosomes were labeled with a yellow (green and red) or red color, respectively. Through the detection and the analysis of the two different fluorescent signals, the autophagic flux was monitored. After culturing the myoblasts for $48 \mathrm{~h}$ with CAPN6 cDNA, decreased red fluorescence was observed in the CAPN6 cDNA groups (CAPN6 group), in comparison with the CRISPR-transfected groups (Control-CRISPR group). This indicated that CAPN6 may decrease autophagy (Fig. 1).

Furthermore, the mechanism responsible for the effects of CAPN6 on autophagy was explored. It was observed that the co-localization of mTOR and LAMP1 proteins (a lysosomal marker) was increased in the CAPN6 overexpression group (CAPN6 group), indicating an increased combination of mTOR and lysosomal degradation. Additionally, the co-localization of mTOR, Raptor and $\alpha$-tubulin (a marker of microtubules) in the CA PN6 group indicated an increased combination of mTORC1 and microtubules. These results indicated that a high CAPN6 expression was positively related 

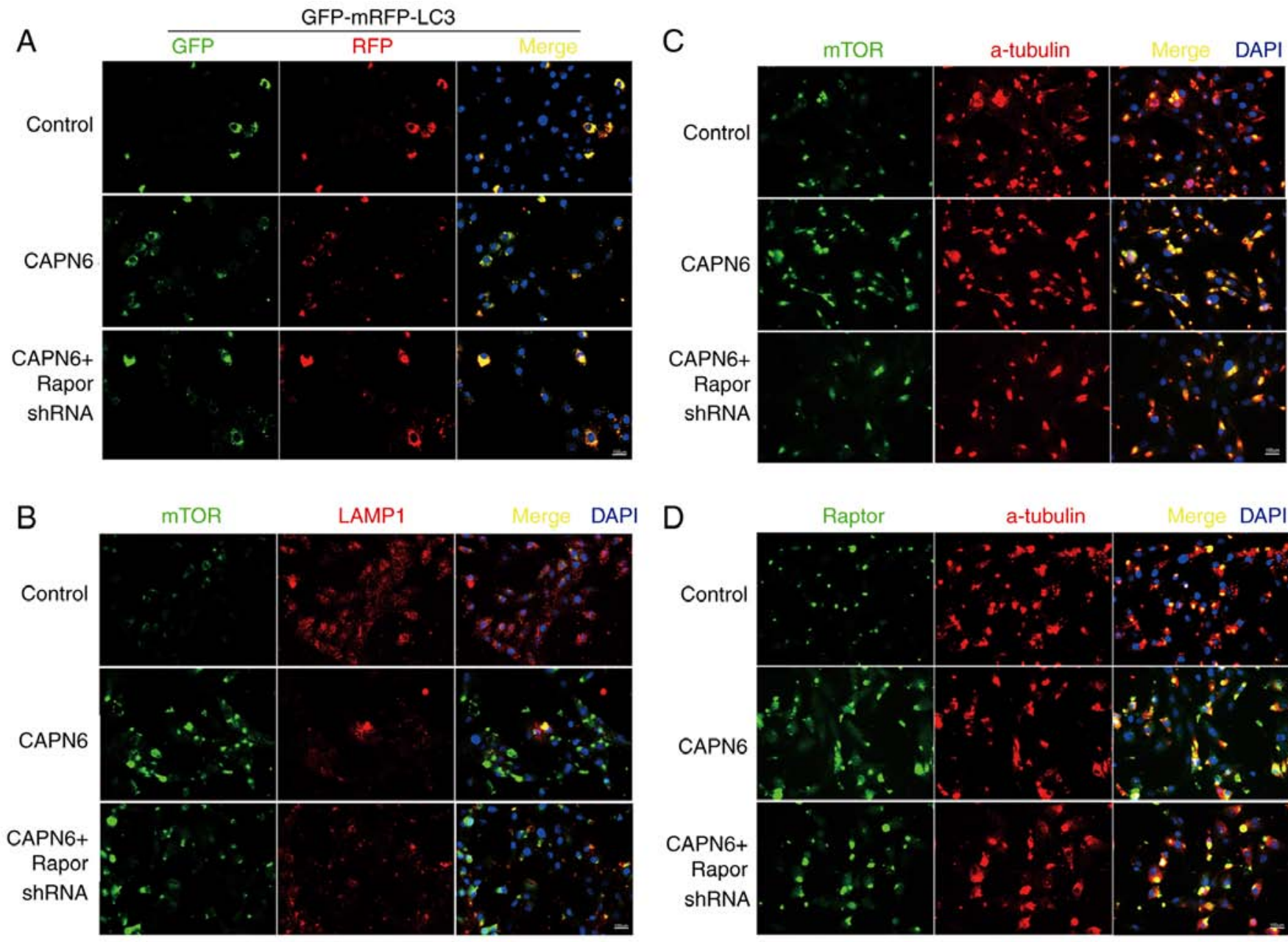

Figure 2. CAPN6 expression leads to mTOR activation. (A) Association between CAPN6 overexpression and changes in GFP-RFP-LC3 fluorescence in CAPN6 and CAPN6 + Raptor shRNA groups. (B-D) Co-localization of mTOR vs. LAMP1, mTOR vs. $\alpha$-tubulin, and raptor vs. $\alpha$-tubulin in the CAPN6 and CAPN6 + Raptor shRNA groups was assessed using immunofluorescence (magnification, x400). CAPN6, calpain 6; GFP, green fluorescence protein; RFP, red fluorescence protein; LC, autophagy-related protein light-chain; CA PN6, CAPN6 cDNA transfection group, CAPN6 overexpression group; CAPN6 + Raptor shRNA, CAPN6 overexpression and Raptor shRNA-transfected group; mTOR, mammalian target of rapamycin; LAMP, lysosomal-associated membrane protein.

to mTORC1 combined with lysosomal and microtubules, and this may be a mechanism through which CAPN6 inhibits autophagy (Fig. 2).

Following a decrease in mTORC1 through transfection with Raptor shRNA, it was revealed that the co-localization of mTOR and LAMP1 proteins, mTOR and $\alpha$-tubulin, Raptor and $\alpha$-tubulin decreased in the CAPN6 overexpression + Raptor shRNA-transfected group (CAPN6 + Raptor shRNA group). In addition, an increase in red fluorescence was observed in the CAPN6 + Raptor shRNA group, as compared with the CAPN6 group. This indicated that mTORC1 combined with lysosomal or microtubules plays an important role in CAPN6 inhibiting autophagy flux (Fig. 2).

Upregulation of CAPN6 by inflammatory cytokines. L6 myoblasts were exposed to $2 \mathrm{ng} / \mathrm{ml} \mathrm{IL-6,} 2 \mathrm{ng} / \mathrm{ml} \mathrm{TNF}-\alpha$, $2 \mathrm{ng} / \mathrm{ml} \mathrm{INF-} \gamma$ and $10 \mathrm{ng} / \mathrm{ml}$ LPS separately and it was demonstrated that the protein expression of CAPN6 increased, particularly in the IFN- $\gamma$-treated L6 myoblasts (Fig. 3). The mRNA levels also increased when the cells were exposed to the four inflammatory agents simultaneously and in a time-dependent manner. The expression levels increased following $6 \mathrm{~h}$ of treatment and were higher at $24 \mathrm{~h}$ (Fig. 3).

CAPN6/mTORC1 activity plays a role in the inhibition of autophagy by inflammatory cytokines. Subsequently, the effects of CAPN6 on autophagy in myoblasts exposed to the four inflammatory agents were assessed. CAPN6 expression significantly increased in the cytokine mixture-treated L6 myoblasts (M group), while the level of LC3II/I decreased significantly. LC3II/I expression increased following CAPN6 knockdown using shRNA in the cytokine mixture-treated L6 myoblasts ( $\mathrm{M}+$ shRNA group; Fig. 4). The autophagic was also examined with fluorescence. It was observed that the fluorescence was not significantly altered in the M group; however, fluorescence increased in the $\mathrm{M}+$ shRNA group, suggesting an increase in the autophagic flux (Fig. 4C).

Finally, the expression and activity of mTORC1 in L6 myoblasts following treatment with the control or cytokine mixture (M group) was evaluated and an increase in mTOR, p-p70s6k1/p70s6k1 and p-4EBP1/4EBP1 expression was observed. Additionally, CAPN6 knockdown (M + shRNA 

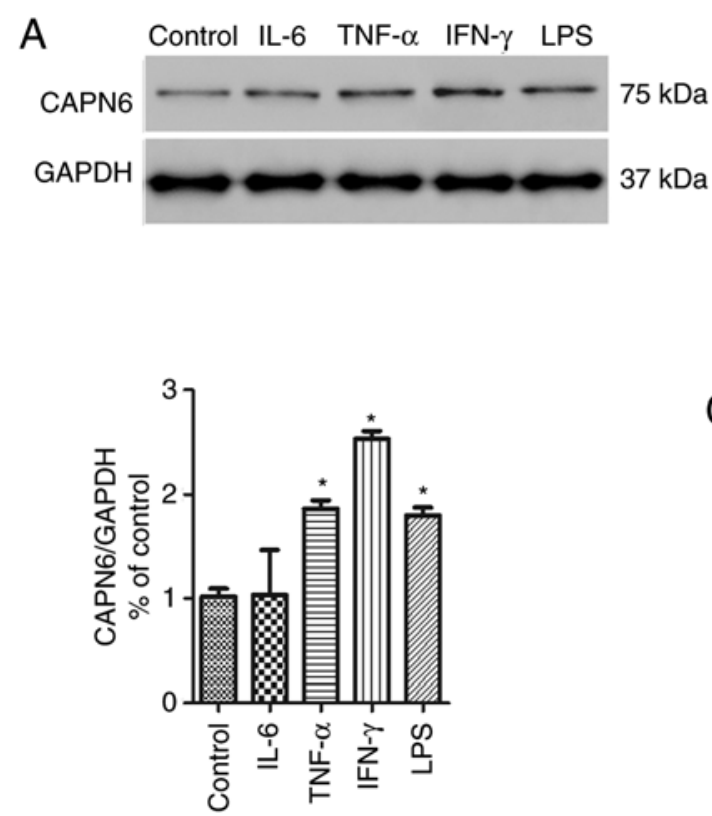

$\mathrm{B}$ 흐
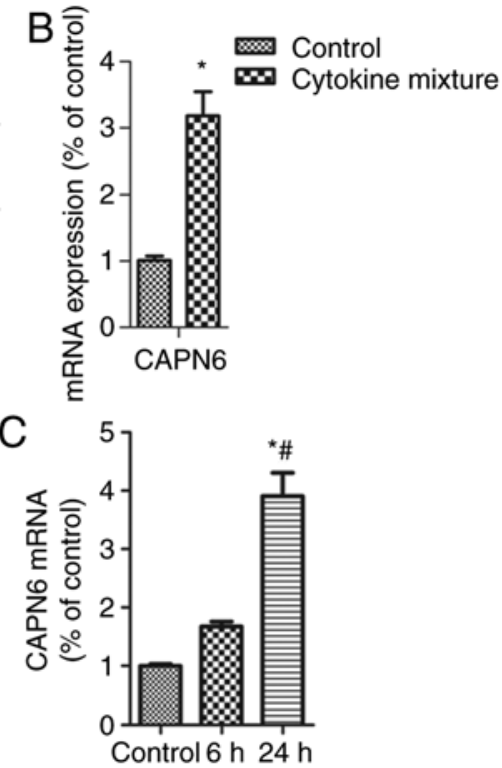
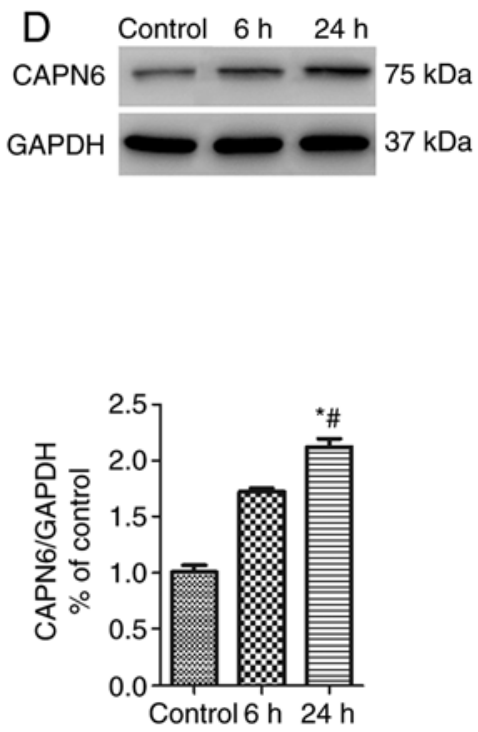

Figure 3. Effects of cytokines on gene expression in L6 myoblasts. (A) L6 myoblasts were grown and treated with $2 \mathrm{ng} / \mathrm{ml} \mathrm{IL-6,} 2 \mathrm{ng} / \mathrm{ml} \mathrm{TNF}-\alpha, 2 \mathrm{ng} / \mathrm{ml}$ INF- $\gamma$ and $10 \mathrm{ng} / \mathrm{ml}$ LPS individually for $24 \mathrm{~h}$, then subjected to western blot analysis. (B) L6 myoblasts were grown and treated with the cytokine mixture for $24 \mathrm{~h}$, then

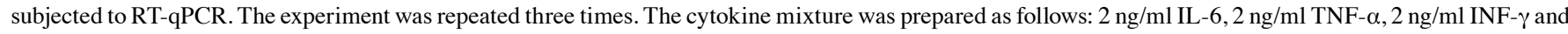
$10 \mathrm{ng} / \mathrm{ml}$ LPS. (C) L6 myoblasts were grown and treated with cytokine mixture for 6 and $24 \mathrm{~h}$, then subjected to RT-qPCR analysis. (D) L6 myoblasts were grown and treated with the cytokine mixture for 6 and $12 \mathrm{~h}$, then subjected to western blot analysis. ${ }^{~} \mathrm{P}<0.05$ as compared with the control group. ${ }^{~} \mathrm{P}<0.05$ in comparison with the 6-h group. IL, interleukin; TNF, tumor necrosis factor; INF, interferon; LPS, lipopolysaccharide.

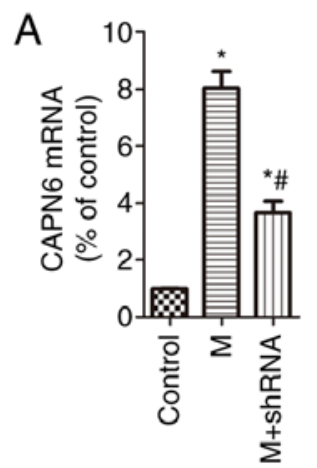

C

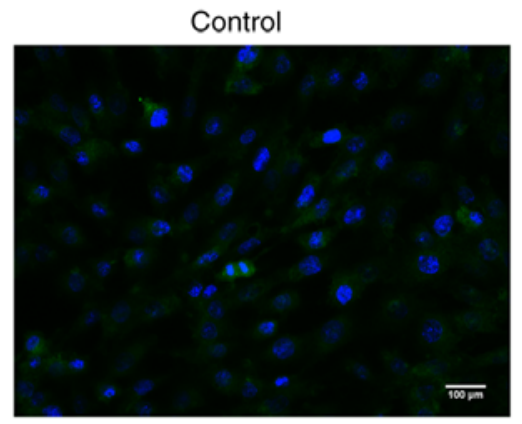

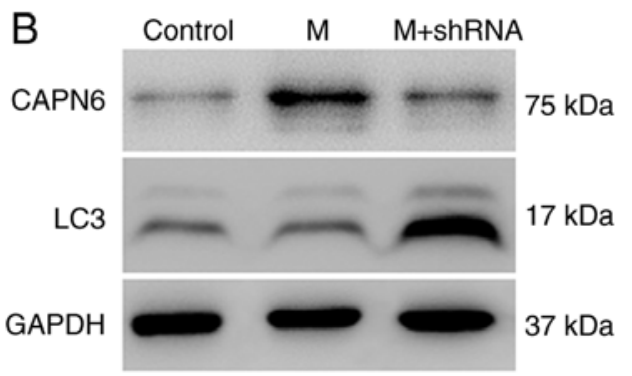

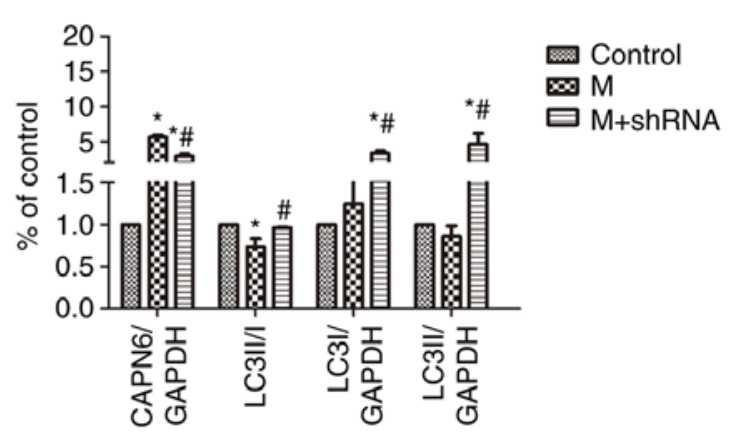

M

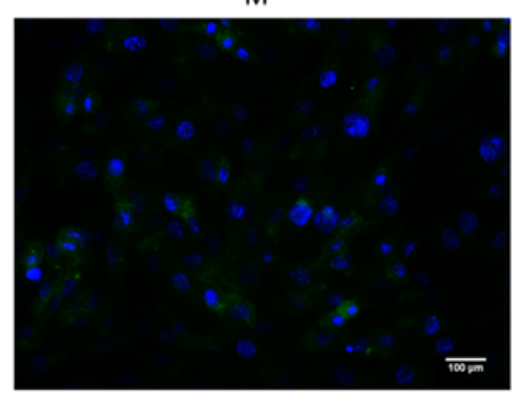

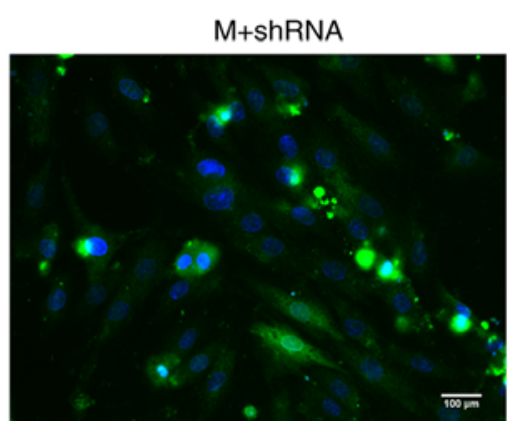

Figure 4. Effects of CAPN6 knockdown on CAPN6 and LC3 expression regulation in rat L6 myoblasts under different conditions. (A) L6 myoblasts were grown, then treated with M (cytokine mixture), M + shRNA (cytokine mixture + transfected CAPN6 shRNA) to assess CAPN6 mRNA expression, evaluated by RT-qPCR. (B) CAPN6 and LC3 protein expression assessed by western blot analysis. (C) LC3 protein expression assessed by immunofluorescence (magnification, $\mathrm{x} 400$ ). The

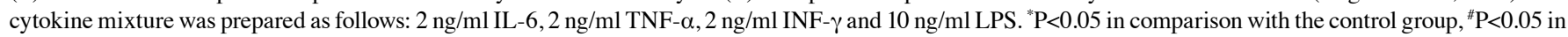
comparison with the M group. CAPN6, calpain 6; LC, autophagy-related protein light-chain; M, cytokine mixture; M + shRNA, cytokine mixture plus sh-CAPN6.

group) culminated in a decrease in protein expression (Fig. 5). However, Raptor expression was not significantly altered in the three groups.
CAPN6 expression is relatively high in CKD. In the in vivo experiments, a rat model of CKD was established. The rats exhibited a decrease in body weight, and reduced muscle mass 


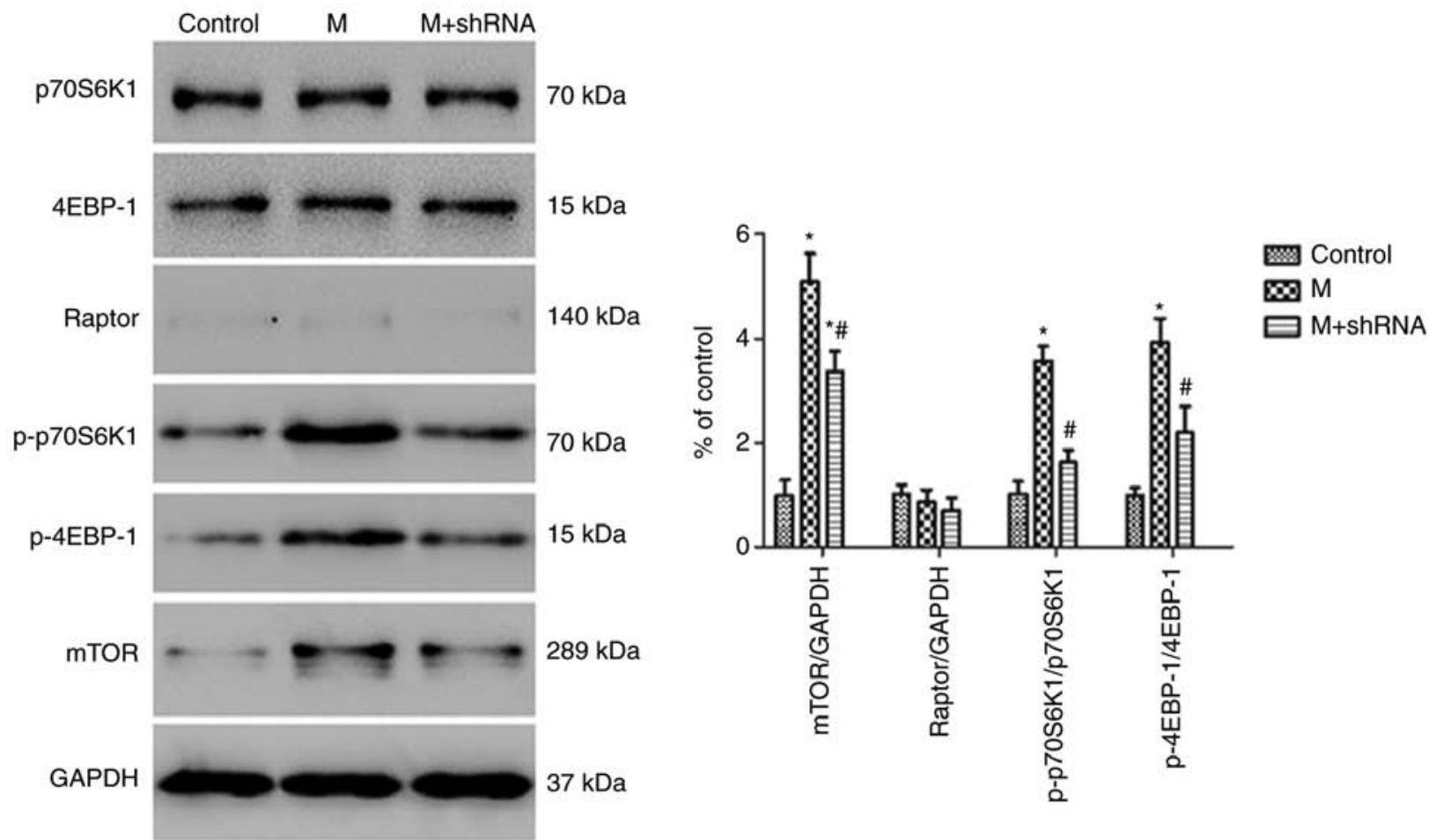

Figure 5. Comparison of indicated biomarker levels under different conditions. Protein expression of mTOR, Raptor, p-p70s6k1/p70s6k1 and p-4EBP1/4EBP1 for the control, M (cytokine mixture) and M + shRNA (cytokine mixture + CAPN6 shRNA-transfected). The cytokine mixture was prepared as follows: $2 \mathrm{ng} / \mathrm{ml} \mathrm{IL-6,} 2 \mathrm{ng} / \mathrm{ml}$ TNF- $\alpha, 2 \mathrm{ng} / \mathrm{ml}$ INF- $\gamma$ and $10 \mathrm{ng} / \mathrm{ml} \mathrm{LPS} .{ }^{*} \mathrm{P}<0.05$ in comparison with the control group, ${ }^{\text {}} \mathrm{P}<0.05$ in comparison with the M group. CAPN6, calpain 6; M, cytokine mixture; $\mathrm{M}+$ shRNA, cytokine mixture + shCAPN6.
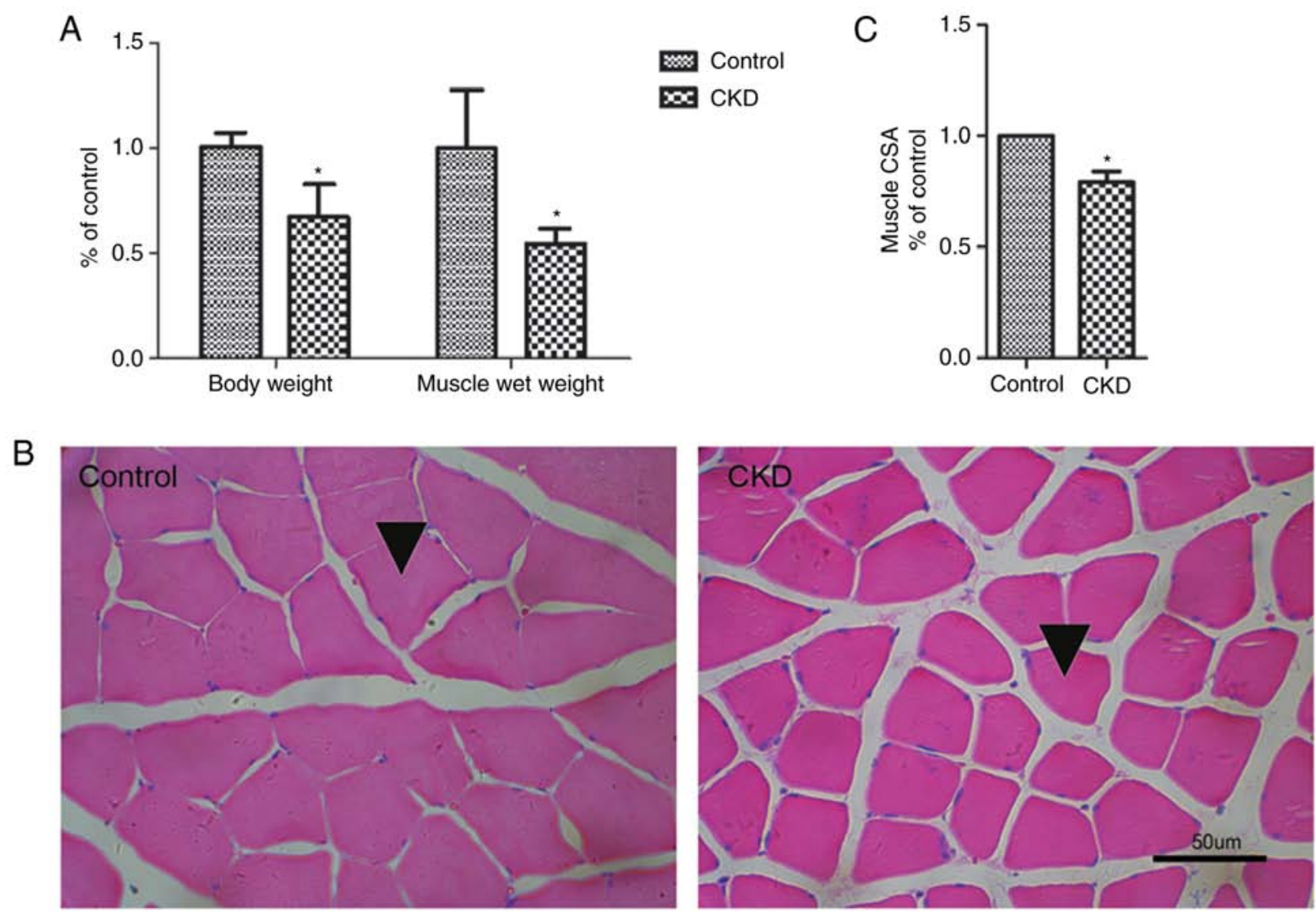

Figure 6. Establishment of the rat model of CKD. Male Sprague-Dawley rats with body weights ranging from 200 to $250 \mathrm{~g}$ were randomly assigned to the CKD or control groups ( $\mathrm{n}=8$, per group). (A) Muscle and body weights of the rats. (B) H\&E staining (magnification, $\mathrm{x} 40$ ). Arrows indicate skeletal muscle fiber. (C) The graph summarizes the CSA of the gastrocnemius muscle in Control and CKD rats. ${ }^{*} \mathrm{P}<0.05$ in comparison with the control group. CKD, chronic kidney disease; H\&E, hematoxylin and eosin; CSA, cross-sectional area. 
Table I. Comparison of serum urea nitrogen, creatinine, albumin, triglyceride and cholesterol levels between the control and CKD group rats.

\begin{tabular}{lrcr}
\hline Variable & \multicolumn{1}{c}{ Control } & CKD & P-value \\
\hline BUN $(\mathrm{mmol} / \mathrm{l})$ & $5.65 \pm 0.94$ & $18.38 \pm 5.49$ & $<0.01$ \\
Cr $(\mu \mathrm{mol} / \mathrm{l})$ & $31.29 \pm 3.97$ & $73.99 \pm 23.50$ & $<0.01$ \\
Alb $(\mathrm{g} / \mathrm{l})$ & $24.22 \pm 2.03$ & $20.67 \pm 4.04$ & 0.027 \\
TC $(\mathrm{mmol} / \mathrm{l})$ & $1.19 \pm 0.07$ & $2.02 \pm 0.28$ & 0.015 \\
TG $(\mathrm{mmol} / \mathrm{l})$ & $2.65 \pm 0.20$ & $0.31 \pm 0.19$ & 0.010 \\
\hline
\end{tabular}

CKD, chronic kidney disease; BUN, blood urea nitrogen; $\mathrm{Cr}$, creatinine; Alb, albumin; TC, total cholesterol; TG, triglyceride.

and muscle CSA (Fig. 6). Blood chemical levels were similar to those similar to those of CKD patients (9), including an increase in BUN, serum creatinine and TC levels, and lower TG and Alb levels (Table I). CAPN6 mRNA and protein expression levels in the gastrocnemius muscles of rats with CKD were also examined, and it was revealed that there was a non-significant increase only (Fig. 7).

\section{Discussion}

In the present study, a novel mechanism, to the best of our knowledge, was revealed, concerning the inhibition of CKD-induced autophagy activation: i) CAPN6 acted with mTOR complex 1 to impair autophagy activity in myoblasts; ii) CAPN6 expression did not increase significantly in the CKD rat muscle atrophy model. Future studies are required, however, to investigate CAPN6 targeting as a novel strategy for the attenuation of CKD-induced skeletal muscle atrophy.

It has been reported that autophagy can help muscle mass maintenance, since the autophagic flux is important for preserving muscle mass and myofiber integrity, and autophagy inhibition is associated with myofiber degeneration and weakness in muscle disorders (40). Additionally, excessive autophagy has been reported to aggravate cell damage through the engulfment of proteins, mitochondria and other organelles, affecting cell health and homeostasis (12). Previous studies have demonstrated that hyperactive autophagy and mitophagy may play important roles in CKD-related muscle atrophy $(9,41)$. In the present study, it was demonstrated that CAPN6 expression impairs autophagy activity in rat L6 myoblasts, whereas CAPN6 knockdown in myoblasts induced autophagy, evidenced by an increase in LC3 expression. As mTORC1 is recruited to the lysosome and is subsequently activated (42), the possible mechanism of CAPN6-induced autophagy inhibition was further explored in the present study. It was demonstrated that mTORC1, combined with lysosomal or microtubules, may play an important role in CAPN6 autophagy flux inhibition.

It has been previously demonstrated that CKD rats exhibit an increase in circulating IL-6 and TNF- $\alpha$ (30,41,43-45). CAPN6 activation and muscle protein wasting can occur in conditions associated with inflammation, as for example in relation to diabetes, acidosis, excess angiotensin II, cancer cachexia and aging. In the present study, it was revealed that CAPN6 knockdown in cytokine-treated myoblasts resulted in an increase in the LC3 expression level. However, LC3

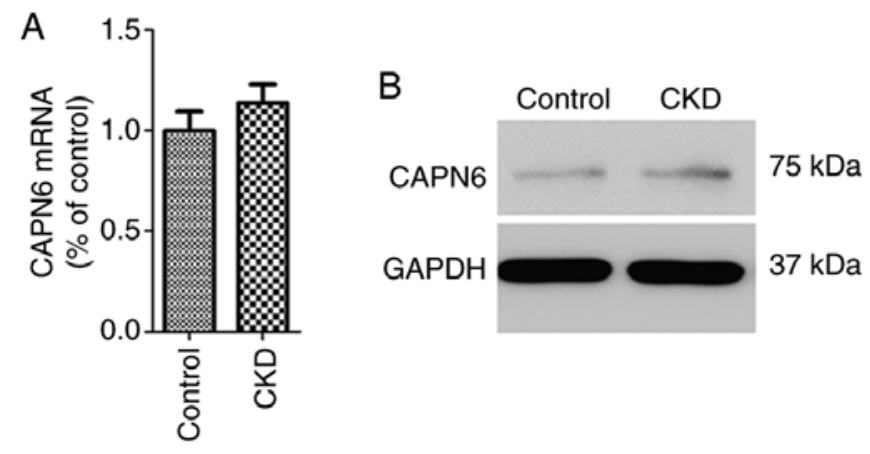

Figure 7. Comparison of CAPN6 expression between Control and CKD rats. (A and B) Changes in the CAPN6 levels in the blood and tissue samples, between the control and CKD group rats. Control group $(n=8)$ and CKD group ( $\mathrm{n}=7)$. CKD, chronic kidney disease; CAPN6, calpain 6.

expression was not significantly altered when CAPN6 expression increased. Indirectly, this may also indicate the role of CAPN6 in autophagy, and it was speculated that an increase in cytokine levels may stimulate CAPN6 expression, in order to alleviate its act on muscle wasting. No evidence was found in support of a significant induction in CAPN6 in atrophic gastrocnemius muscles in a rat model of CKD; thus, it was speculated that there may be other factors inhibiting CAPN6 expression in CKD, including fasting, insulin/insulin growth factor-1 (IGF-1) signal (46), glucocorticoids (47) and inflammation (48), that may also influence autophagy in vivo.

In a previous study, it was reported that CAPN6 deficiency promoted embryonic skeletal muscle development (16). However, CAPN6 expression was induced in adult mouse skeletal muscle regeneration following cardiotoxin treatment, indicating that CAPN6 was able to inhibit adult mouse skeletal muscle differentiation (16). In fact, muscle regeneration, in response to various stimuli or injuries, will activate quiescent muscle stem cells and cause their differentiation into the myotube and myofiber cells, accordingly (49). Another study revealed that CAPN6 mRNA was exclusively expressed in developing skeletal and heart muscles, although it was also observed in specific types of cells in the lungs, kidneys, and placenta and various epithelial cell types (50). This was indicative of the role of CAPN6 in muscle development and regeneration. Taken together, it can be hypothesized that CAPN6 may inhibit skeletal muscle differentiation and regeneration through autophagy suppression. 
In conclusion, the present study demonstrated that CAPN6 overexpression may downregulate autophagy. However, the balance concerning its detrimental vs. protective roles warrants further elucidation, particularly through in vivo studies. The present study was a proof-of-principle study, and did not present data concerning the evaluation of autophagy marker levels in the gastrocnemius muscles and the canonical mTOR/ULK1/2 and AMPK pathways regulating autophagy. Therefore, further studies are required in order to confirm the role of CAPN6 in the regulation of autophagy in CKD-induced skeletal muscle atrophy.

\section{Acknowledgements}

Not applicable.

\section{Funding}

The present study was supported in part by a grant from the Shanghai Youth Science and Technology Excellence Plan Project 2019 (grant no. 19YF1439100), and in part by the National Natural Science Foundation of China (grant no. 81900671).

\section{Availability of data and materials}

The analyzed datasets generated during this study are available from the corresponding author on reasonable request.

\section{Authors' contributions}

YYZ and LJG contributed to the acquisition, analysis and interpretation of all data, and to the drafting of the manuscript. JSJ and NZ analyzed and interpreted the data, and revised the manuscript. LW and MCC contributed to the conception, analysis and interpretation of the data. SR and WJY contributed to the study conception and design, and confirm the authenticity of all the raw data. All the authors have read and approved the final version of the manuscript for submission.

\section{Ethics approval and consent to participate}

All animal protocols were approved by the Animal Care and Use Committee of Shanghai Jiao Tong University (Shanghai, China) and followed the Guidelines of the Care and Use of Laboratory Animals issued by the Chinese Council on Animal Research (project no. 2015DW001).

\section{Patient consent for publication}

Not applicable.

\section{Competing interests}

The authors declare that they have no competing interests.

\section{References}

1. Centers for Disease Control and Prevention (CDC). Prevalence of chronic kidney disease and associated risk factors-United States, 1999-2004. MMWR. MMWR Morb Mortal Wkly Rep 56: 161-165, 2007.
2. Schardong J, Marcolino MAZ and Plentz RDM: Muscle atrophy in chronic kidney disease. Adv Exp Med Biol 1088: 393-412, 2018.

3. Thome T, Salyers ZR, Kumar RA, Hahn D, Berru FN, Ferreira LF, Scali ST and Ryan TE: Uremic metabolites impair skeletal muscle mitochondrial energetics through disruption of the electron transport system and matrix dehydrogenase activity. Am J Physiol Cell Physiol 317: C701-C713, 2019.

4. Garg AX, Blake PG, Clark WF, Clase CM, Haynes RB and Moist LM: Association between renal insufficiency and malnutrition in older adults: Results from the NHANES III. Kidney Int 60: 1867-1874, 2001.

5. Fouque D, Kalantar-Zadeh K, Kopple J, Cano N, Chauveau P, Cuppari L, Franch H, Guarnieri G, Ikizler TA, Kaysen G, et al: A proposed nomenclature and diagnostic criteria for protein-energy wasting in acute and chronic kidney disease. Kidney Int 73: 391-398, 2008.

6. Carrero JJ, Stenvinkel P, Cuppari L,Ikizler TA, Kalantar-Zadeh K, Kaysen G, Mitch WE, Price SR, Wanner C, Wang AY, et al: Etiology of the protein-energy wasting syndrome in chronic kidney disease: A consensus statement from the International Society of Renal Nutrition and Metabolism (ISRNM). J Ren Nutr 23: 77-90, 2013.

7. Kovesdy CP, Kopple JD and Kalantar-Zadeh K: Management of protein-energy wasting in non-dialysis-dependent chronic kidney disease: Reconciling low protein intake with nutritional therapy. Am J Clin Nutr 97: 1163-1177, 2013.

8. Kanazawa Y, Nakao T, Murai S, Okada T and Matsumoto $\mathrm{H}$ : Diagnosis and prevalence of protein-energy wasting and its association with mortality in Japanese haemodialysis patients. Nephrology (Carlton) 22: 541-547, 2017.

9. Zhang YY, Gu LJ, Huang J, Cai MC, Yu HL, Zhang W, Bao JF and Yuan WJ: CKD autophagy activation and skeletal muscle atrophy-a preliminary study of mitophagy and inflammation. Eur J Clin Nutr 73: 950-960, 2019.

10. Kobayashi S: Choose delicately and reuse adequately: The newly revealed process of autophagy. Biol Pharm Bull 38: 1098-1103, 2015.

11. Klionsky DJ: Autophagy revisited: A conversation with Christian de Duve. Autophagy 4: 740-743, 2008.

12. Mizushima $\mathrm{N}$ and Komatsu M: Autophagy: Renovation of cells and tissues. Cell 147: 728-741, 2011.

13. Demarchi F, Bertoli C, Copetti T, Eskelinen EL and Schneider C: Calpain as a novel regulator of autophagosome formation. Autophagy 3: 235-237, 2007.

14. Dear N, Matena K, Vingron M and Boehm T: A new subfamily of vertebrate calpains lacking a calmodulin-like domain: Implications for calpain regulation and evolution. Genomics 45: 175-184, 1997.

15. Tonami K, Kurihara Y, Aburatani H, Uchijima Y, Asano T and Kurihara H: Calpain 6 is involved in microtubule stabilization and cytoskeletal organization. Mol Cell Biol 27: 2548-2561, 2007.

16. Tonami K, Hata S, Ojima K, Ono Y, Kurihara Y, Amano T, Sato T, Kawamura Y, Kurihara H and Sorimachi H: Calpain-6 deficiency promotes skeletal muscle development and regeneration. PLoS Genet 9: e1003668, 2013.

17. Guttula SV, Rao AA, Sridhar GR, Chakravarthy MS, Nageshwararo K and Rao P: Cluster analysis and phylogenetic relationship in biomarker identification of type 2 diabetes and nephropathy. Int J Diabetes Dev Ctries 30: 52-56, 2010.

18. Ko YA, Mohtat D, Suzuki M, Park AS, Izquierdo MC, Han SY, Kang HM, Si H, Hostetter T, Pullman JM, et al: Cytosine methylation changes in enhancer regions of core pro-fibrotic genes characterize kidney fibrosis development. Genome Biol 14: R108, 2013.

19. Andrique C, Morardet L, Linares LK, Cissé MY, Merle C, Chibon F, Provot S, Haÿ E, Ea HK, Cohen-Solal M and Modrowski D: Calpain-6 controls the fate of sarcoma stem cells by promoting autophagy and preventing senescence. JCI insight 3: e121225, 2018.

20. Corona Velazquez AF and Jackson WT: So many roads: The multifaceted regulation of autophagy induction. Mol Cell Biol 38: e00303-18, 2018.

21. Zhang C, Yang L, Geng YD, An FL, Xia YZ, Guo C, Luo JG, Zhang LY, Guo QL and Kong LY: Icariside II, a natural mTOR inhibitor, disrupts aberrant energy homeostasis via suppressing mTORC1-4E-BP1 axis in sarcoma cells. Oncotarget 7: 27819-27837, 2016.

22. Morita M, Gravel SP, Hulea L, Larsson O, Pollak M, St-Pierre J and Topisirovic I: mTOR coordinates protein synthesis, mitochondrial activity and proliferation. Cell Cycle 14: 473-480, 2015. 
23. Francipane MG and Lagasse E: mTOR pathway in colorectal cancer: An update. Oncotarget 5: 49-66, 2014.

24. Xu J, Wu Y, Lu G, Xie S, Ma Z, Chen Z, Shen HM and Xia D: Importance of ROS-mediated autophagy in determining apoptotic cell death induced by physapubescin B. Redox Biol 12 : 198-207, 2017.

25. Wullschleger $\mathrm{S}$, Loewith $\mathrm{R}$ and Hall MN: TOR signaling in growth and metabolism. Cell 124: 471-484, 2006.

26. Hands SL, Proud CG and Wyttenbach A: mTOR's role in ageing: Protein synthesis or autophagy? Aging 1: 586-597, 2009.

27. Liu Y, Mei C, Sun L, Li X, Liu M, Wang L, Li Z, Yin P, Zhao C, Shi Y, et al: The PI3K-Akt pathway regulates calpain 6 expression, proliferation, and apoptosis. Cell Signal 23: 827-836, 2011.

28. Szeto CC, Kwan BC, Chow KM, Lai KB, Chung KY, Leung CB and Li PK: Endotoxemia is related to systemic inflammation and atherosclerosis in peritoneal dialysis patients. Clin J Am Soc Nephrol 3: 431-436, 2008.

29. McIntyre CW, Harrison LE, Eldehni MT, Jefferies HJ, Szeto CC, John SG, Sigrist MK, Burton JO, Hothi D, Korsheed S, et al Circulating endotoxemia: A novel factor in systemic inflammation and cardiovascular disease in chronic kidney disease. Clin J Am Soc Nephrol 6: 133-141, 2011.

30. Thomas SS, Dong Y, Zhang L and Mitch WE: Signal regulatory protein-alpha interacts with the insulin receptor contributing to muscle wasting in chronic kidney disease. Kidney Int 84 308-316, 2013.

31. Wang DT, Lu L, Shi Y, Geng ZB, Yin Y, Wang M and Wei LB: Supplementation of ketoacids contributes to the up-regulation of the Wnt7a/Akt/p70S6K pathway and the down-regulation of apoptotic and ubiquitin-proteasome systems in the muscle of $5 / 6$ nephrectomised rats. Br J Nutr 111: 1536-1548, 2014.

32. Bigelman E, Cohen L, Aharon-Hananel G, Levy R, Rozenbaum Z, Saada A, Keren G and Entin-Meer M: Pathological presentation of cardiac mitochondria in a rat model for chronic kidney disease. PLoS One 13: e0198196, 2018.

33. Rempel LCT, Faustino VD, Foresto-Neto O, Fanelli C, Arias SCA, Moreira GCDS, Nascimento TF, Ávila VF, Malheiros DMAC Câmara NOS, et al: Chronic exposure to hypoxia attenuates renal injury and innate immunity activation in the remnant kidney model. Am J Physiol Renal Physiol 317: F1285-F1292, 2019.

34. Close B, Banister K, Baumans V, Bernoth EM, Bromage N, Bunyan J, Erhardt W, Flecknell P, Gregory N, Hackbarth H, et al: Recommendations for euthanasia of experimental animals: Part 1. DGXI of the European Commission. Lab Anim 30: 293-316, 1996

35. Wu Y, Zhao W, Zhao J, Zhang Y, Qin W, Pan J, Bauman WA Blitzer RD and Cardozo C: REDD1 is a major target of testosterone action in preventing dexamethasone-induced muscle loss. Endocrinology 151: 1050-1059, 2010.

36. Qin W, Pan J, Wu Y, Bauman WA and Cardozo C: Anabolic steroids activate calcineurin-NFAT signaling and thereby increase myotube size and reduce denervation atrophy. Mol Cell Endocrinol 399: 336-345, 2015.

37. Livak KJ and Schmittgen TD: Analysis of relative gene expression data using real-time quantitative PCR and the 2(-Delta Delta C(T)) method. Methods 25: 402-408, 2001
38. Xu B, Bai B, Sha S, Yu P, An Y, Wang S, Kong X, Liu C, Wei N, Feng Q and Zhao Q: Interleukin-1 $\beta$ induces autophagy by affecting calcium homeostasis and trypsinogen activation in pancreatic acinar cells. Int J Clin Exp Pathol 7: 3620-3631, 2014.

39. Kimura S, Noda T and Yoshimori T: Dissection of the autophagosome maturation process by a novel reporter protein, tandem fluorescent-tagged LC3. Autophagy 3: 452-460, 2007.

40. Masiero E, Agatea L, Mammucari C, Blaauw B, Loro E, Komatsu M, Metzger D, Reggiani C, Schiaffino S and Sandri M: Autophagy is required to maintain muscle mass. Cell Metabol 10: 507-515, 2009.

41. Zhang YY, Huang J, Yang M, Gu LJ, Ji JY, Wang LJ and Yuan WJ: Effect of a low-protein diet supplemented with keto-acids on autophagy and inflammation in 5/6 nephrectomized rats. Biosci Rep 35: e00263, 2015.

42. Sancak Y, Bar-Peled L, Zoncu R, Markhard AL, Nada S and Sabatini DM: Ragulator-Rag complex targets mTORC1 to the lysosomal surface and is necessary for its activation by amino acids. Cell 141: 290-303, 2010.

43. Du J, Mitch WE, Wang X and Price SR: Glucocorticoids induce proteasome $\mathrm{C} 3$ subunit expression in L6 muscle cells by opposing the suppression of its transcription by NF-kappa B. J Biol Chem 275: 19661-19666, 2000.

44. Cai D, Yuan M, Frantz DF, Melendez PA, Hansen L, Lee J and Shoelson SE: Local and systemic insulin resistance resulting from hepatic activation of IKK-beta and NF-kappaB. Nat Med 11: 183-190, 2005.

45. Zhang L, Rajan V, Lin E, Hu Z, Han HQ, Zhou X, Song Y, Min H, Wang X, Du J and Mitch WE: Pharmacological inhibition of myostatin suppresses systemic inflammation and muscle atrophy in mice with chronic kidney disease. FASEB J 25: 1653-1663, 2011.

46. Zhao J, Brault JJ, Schild A, Cao P, Sandri M, Schiaffino S, Lecker SH and Goldberg AL: FoxO3 coordinately activates protein degradation by the autophagic/lysosomal and proteasomal pathways in atrophying muscle cells. Cell Metabol 6: 472-483, 2007.

47. Yamamoto D, Maki T, Herningtyas EH, Ikeshita N, Shibahara H, Sugiyama Y, Nakanishi S, Iida K, Iguchi G, Takahashi Y, et al: Branched-chain amino acids protect against dexamethasone-induced soleus muscle atrophy in rats. Muscle Nerve 41: 819-827, 2010.

48. Bailey JL, Zheng B, Hu Z, Price SR and Mitch WE: Chronic kidney disease causes defects in signaling through the insulin receptor substrate/phosphatidylinositol 3-kinase/Akt pathway: Implications for muscle atrophy. J Am Soc Nephrol 17: 1388-1394, 2006.

49. Turner NJ and Badylak SF: Regeneration of skeletal muscle. Cell Tissue Res 347: 759-774, 2012

50. Dear TN and Boehm T: Diverse mRNA expression patterns of the mouse calpain genes Capn5, Capn6 and Capn11 during development. Mech Dev 89: 201-209, 1999.

This work is licensed under a Creative Commons Attribution-NonCommercial-NoDerivatives 4.0 International (CC BY-NC-ND 4.0) License. 